\title{
Assessement of angiogenesis reveals blood vessel heterogeneity in lung carcinoma
}

\author{
AMALIA BIRAU ${ }^{1}$, RALUCA AMALIA CEAUSU ${ }^{2}$, ANCA MARIA CIMPEAN ${ }^{2}$, \\ PUSA GAJE $^{2}$, MARIUS RAICA ${ }^{2}$ and TEODORA OLARIU ${ }^{1}$ \\ ${ }^{1}$ Department of Intensive Care, 'Vasile Goldis' Western University of Arad, Arad, Arad 310396; ${ }^{2}$ Department of Histology, \\ 'Victor Babeș' University of Medicine and Pharmacy, Angiogenesis Research Center, \\ Timisoara, Timis 300041, Romania
}

Received April 16, 2012; Accepted July 24, 2012

DOI: $10.3892 / \mathrm{ol} .2012 .893$

\begin{abstract}
Despite advances in treatment, the prognosis for lung cancer patients remains poor. Angiogenesis appears to be a promising target for lung cancer therapy; however, the clinical significance of vascular changes are not completely understood. The aim of this study was to evaluate the types and morphology of blood vessels in various lung carcinomas. Using double immunostaining, we investigated 39 biopsies from patients admitted with various histological types of lung carcinoma. Tumor blood vessels were quantified separately for CD34/smooth muscle actin and described as either immature, intermediate or mature. Double immunostaining evaluation of the type of blood vessels in lung carcinomas revealed a marked heterogeneity. The immature and intermediate type of vessels were more common in adenocarcinomas (ADCs) and squamous cell carcinomas (SCCs) of the lung. Small cell lung carcinomas revealed a significant correlation between pathological and immature types of blood vessels. Therefore, quantifying the types of tumor vessels in lung carcinomas may be an important element to improve the results of anti-vascular therapy.
\end{abstract}

\section{Introduction}

Lung cancer affects more individuals worldwide than any other type of cancer, and is the leading cause of cancer mortality in males and females. The global incidence of this disease is rising by $0.5 \%$ per year, and the number of cancer mortalities caused by lung cancer is expected to increase by up to $50 \%$ by 2020 (1).

Correspondence to: Dr Anca Maria Cimpean, Department of Histology, 'Victor Babeș' University of Medicine and Pharmacy, Angiogenesis Research Center, 2 Piata Eftimie Murgu, Timisoara, Timis 300041, Romania

E-mail: ancacimpean1972@yahoo.com

Key words: angiogenesis, lung carcinoma, blood vessels, double immunostaining
Formation of the pulmonary circulation has been described as a process that depends on two basic mechanisms: vasculogenesis and angiogenesis. The distal vasculature arises by vasculogenesis (embryonic day nine in the mouse model) (2) and the proximal vasculature arises by angiogenesis (starts at approximately embryonic day 12); however, this theory remains controversial (3).

Angiogenesis, the formation of new blood vessels, is essential for tumor growth and metastasis (4). A total of six tumor vessel types have been identified. Four vessel types (mother vessels, capillaries, glomeruloid microvascular proliferations and vascular malformations) develop from preexisting normal venules and capillaries, and two vessel types (feeder arteries and draining veins) develop from arterio-venogenesis, a parallel process that involves the remodeling of preexisting arteries and veins (5). There are several well-known mechanisms of blood vessel formation in normal and tumor tissues, including sprouting angiogenesis, vasculogenesis, intussusception, vessel co-option, vasculogenic mimicry and tumor cell-endothelial cell transdifferentiation (6). The new vascular network is considered 'immature' and varies from normal (or mature) vascular structures. Immature vessels lack the normal vascular network organization, are irregularly shaped, are composed of abnormal basement membranes and pericytes, and have an increased permeability.

Microvessel pericyte coverage index revealed intermediate values in lung and prostate carcinomas, high values in mammary and colon carcinomas, and low values in glioblastomas and renal cell carcinomas (7). Passalidou et al (8) described a group of non-small cell lung carcinomas (NSCLC) without morphological evidence of neoangiogenesis. In these tumors, the vascular phenotype was that of normal vessels and there was no neoangiogenesis. Following this, the vascular architecture in lung adenocarcinomas (ADCs) was classified into three types of patterns: diffuse, alveolar (nonangiogenic) and mixed (9). Kakolyris et al (10) demonstrated that there is a large variation in the level of differentiation of tumor vasculature in lung carcinoma subtypes. The authors suggested that capillary maturation may be correlated with microvessel number, improving the identification of patients who may benefit from specific antiangiogenic therapies. 
Despite advances in treatment, the five-year survival rate does not exceed 15\% (11). In 2006, bevacizumab was approved for first-line treatment of advanced, non-squamous NSCLC to be administered in combination with platinium-based chemotherapy (12). Nevertheless, the prognosis for patients with lung cancer remains poor; thus, quantifying the types of blood vessels may aid the improvement of lung cancer therapy.

The aim of this study was to use double immunostaining methods to evaluate the types and morphology of blood vessels in various types of lung carcinomas.

\section{Materials and methods}

In our study, we included 39 biopsies from patients with various types of lung carcinoma. Sections $(5 \mu \mathrm{m})$ were fixed in buffered formalin and embedded in paraffin. For pathological diagnosis, slides were stained with haematoxylin and eosin (H\&E). For double immunostainings, CD34 (QBEnd10 clone; dilution, 1:25) was applied for 30 min and smooth muscle actin (SMA; clone 1A4; ready to use) for $30 \mathrm{~min}$. The EnVision Doublestain kit was used as a visualization system (DakoCytomation; Glostrup, Denmark). All reagents were purchased from Dako Cytomation. The entire immunohistochemical procedure was developed with a Dako Cytomation Autostainer (Dako Cytomation). Tumor blood vessels were quantified separately for CD34/SMA according to Gee et al (13) as either immature, intermediate or mature. We used the 'hot spot' method to evaluate the total number of vessels, which were then separately counted in the same field and defined as immature (CD34 ${ }^{+} / \mathrm{SMA}^{-}$, without lumen), intermediate $\left(\mathrm{CD} 34^{+}\right.$, with perfused lumen and with negative or weak positive reaction for SMA) or mature vessels $\left(\mathrm{CD} 34^{+} / \mathrm{SMA}^{+}\right)$. Microscopic images were captured and processed using Nikon Lucia G software (Nikon, Tokyo, Japan).

This study was approved by the local research ethics committee of 'Victor Babes' University of Medicine and Pharmacy Timisoara, Romania, and informed consent was obtained from all subjects according to the World Medical Association Declaration of Helsinki.

\section{Results}

Pathological evaluation. Pathological evaluation of lung cancer specimens revealed seven cases of small cell carcinomas, five cases of ADC, one case of large cell lung carcinoma (LCLC), one case of hepatoid carcinoma and 25 cases of squamous cell carcinoma (SCC).

Double immunostaining. Double immunostaining revealed tumor blood vessel heterogeneity in the same and different pathological subtypes of lung carcinoma. Vessels from peritumoral and intratumoral areas varied in size, were irregular in shape and contained numerous branches. The vessels were arranged among the tumor cells with relatively uniform distribution. We identified a high level of variability in terms of blood vessel morphology. Vessels presented with variable dimensions, irregular and narrow lumens, and intense branching character. The presence of cords and isolated endothelial cells suggests the possibility of the sprouting angiogenesis mechanism. In the tumor area, we occasionally identified vessels with a large
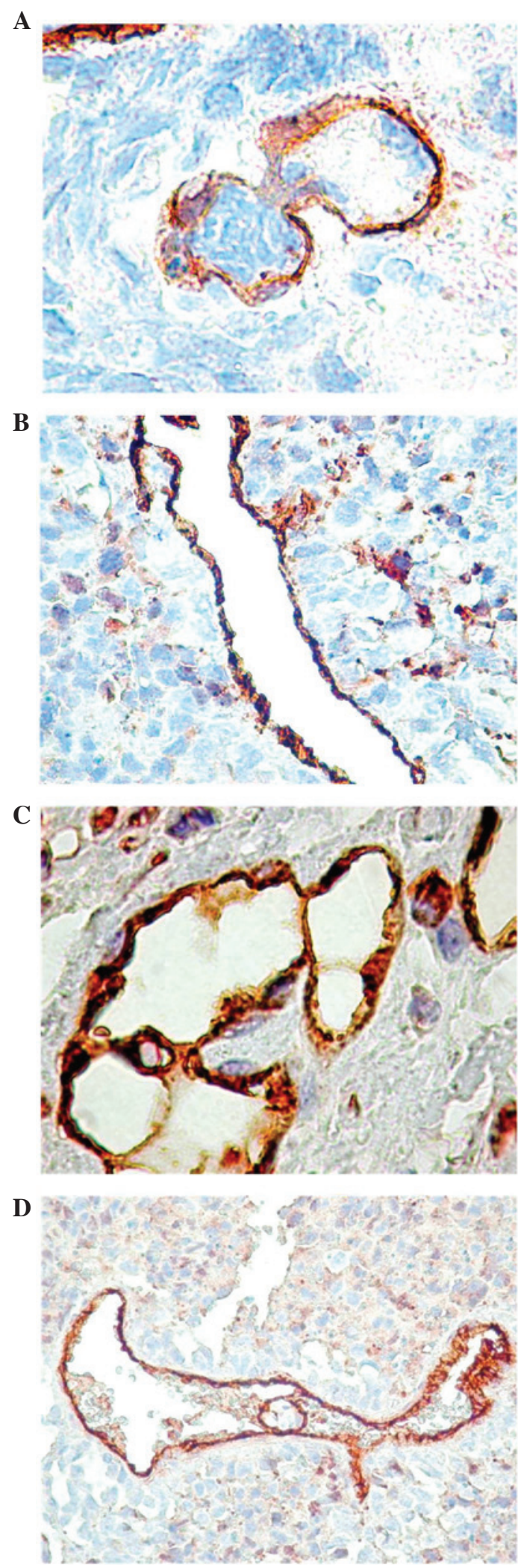

Figure 1. CD34/SMA double immunostaining. (A) Intussusceptions phenomenon (magnification, $\mathrm{x} 400$ ). (B) Vessels from the peritumoral area (magnification, x100). (C) Glomeruloid bodies (magnification, x400). (D) Vessel-like structures with numerous tumor emboli inside blood vessels (magnification, x100). SMA, smooth muscle actin.

lumen and prominent endothelium, suggesting the possibility of the intussusception mechanism (Fig. 1A). This process is considered to arise from preexisting blood vessels during 
tumor proliferation. In the peritumoral area, the vessels were composed of a large lumen, relatively regular shape and were usually surrounded by inflammatory infiltrate (Fig. 1B).

Microvascular density (MVD). The mean MVD value of the vessels was 31.86 for ADCs, 23.28 for small cell carcinomas, 16 for LCLCs, 23 for hepatoid carcinomas and 23.99 for SCCs. The highest and lowest MVD values were identified in lung ADCs and LCLCs, respectively. In all cases, the immature and intermediate types of blood vessels were predominant with a few differences associated with pathological subtypes. No correlation was identified between the pathological type and the total number of blood vessels $(\mathrm{P}=0.357)$. We identified a significant correlation between the pathological type and the number of immature $(\mathrm{P}=0.038)$ and mature vessels $(\mathrm{P}=0.036)$; however, no correlation was identified between the pathological type and the number of intermediate type vessels $(\mathrm{P}=0.447)$.

Lung $A D C$. The average number of the immature (16.66) and the intermediate (14.06) vessels demonstrated almost similar values compared to the average number of mature vessels (1.19) from lung ADCs. These observations are supported by the significant correlation identified between the total number of vessels and the number of immature vessels $(\mathrm{P}=0.039)$ from this pathological type. In the ADC specimens, immature vessels predominated, except in one case where we revealed the presence of intussusceptions and a high number of CD34 structures without SMA expression, suggesting the formation of glomeruloid bodies (Fig. 1C).

Small cell lung carcinoma. Immature blood vessels were the predominant type observed in small cell lung carcinoma samples. This was supported by the correlation between immature and pathological vessels $(\mathrm{P}=0.011)$. The pathological type did not correlate with intermediate and mature types of vessels $(\mathrm{P}=0.151$ and $\mathrm{P}=0.405$, respectively).

SCC. A significant correlation between the total number of vessels and the number of immature vessels $(\mathrm{P}=0.005)$ was identified in lung SCC samples. A representative correlation with intermediate type blood vessels $(\mathrm{P}=0.018)$ was also revealed; however, there was no correlation with mature type blood vessels $(\mathrm{P}=0.512)$.

$L C L C$. A high number of $\mathrm{SMA}^{+}$tumor cells was observed in the tumor tissues and around the blood vessels in the LCLC samples. Vessel-like structures containing numerous tumor emboli were also detected in the blood vessels (Fig. 1D).

\section{Discussion}

Malignant human tumors are characterized by varying degrees of angiogenesis and pericyte recruitment. The degree of angiogenesis in human tumors varies and may be extremely low in certain types of tumors. The suitability of tumors for antiangiogenic therapies may differ between various tumor types or within one type of tumor.

Maeda et al (14) examined the association between the number of circulating endothelial progenitor cells (EPCs) and intratumoral MVD, both of which may be markers for neovascularization, as well as the various lung cancer histological types, particularly ADC. They revealed no statistically significant differences in the number of EPCs or the MVD value between the ADC and SCC subtypes. Among the ADC histological subtypes, a higher number of EPCs and a greater MVD value was identified, which was significantly more frequent in solid ADCs compared with non-solid. These patients may be the best candidates for antiangiogenic therapies.

Dagnon et al (15) analyzed the distance between cancer cells, blood vessels and the microvasculature organization in NSCLC in comparison with SCC and ADC. This computerized morphometric study revealed a significantly higher MVD value in ADCs compared with SCCs, particularly close to the invading edge. We also identified similar mean values for the number of immature, intermediate and mature vessels in the lung SCC and ADC subtypes.

Due to the continuous and excessive synthesis of the vascular endothelial growth factor (VEGF) in cancer tissue, tumor vessels remain immature and lack the tight association between mural cells and endothelial tubes. The immature tumor vessels display high vascular permeability; thus, the tumor tissue is edematous, containing extravasated plasma components. In addition to edema, the expansion of cancer tissue results in increased interstitial pressure, causing impaired tumor blood flow (16).

Antiangiogenic therapy with bevacizumab, an anti-VEGF antibody, predominantly targets immature blood vessels. Zhao et al (17) demonstrated that there are two major types of microvessels in lung cancer vasculature, undifferentiated and differentiated. The MVD value of undifferentiated vessels is a favorable predictor for patients with NSCLC treated with a chemotherapy regimen and bevacizumab, with a higher MVD value correlating with a better treatment response. Further studies are required to verify the predictive role of MVD in the treatment of NSCLC with bevacizumab. We identified that immature and intermediate vessel types are predominantly expressed in lung SCCs and ADCs, while immature vessel types are predominantly expressed in small cell lung carcinoma.

One of the major problems associated with bevacizumab therapy is the exclusion of patients with brain metastases and/or squamous histology who are receiving this therapeutic option as they represent a significant proportion of the advanced NSCLC patient population (18). In our study, we revealed a significant correlation between the total number of vessels in the lung SCCs and the number of immature and intermediate type vessels; however, no correlation was identified with mature type vessels. Jubb et al (19) demonstrated that the proliferation index, VEGF expression, MVD and the number of mature vessels were discordant between primary and secondary cancers.

In conclusion, double immunostaining evaluation of the types of blood vessels in lung carcinoma demonstrated a marked heterogeneity. The highest MVD was identified in lung ADCs and the lowest in LCLCs. The immature and intermediate types of vessels were more common in ADCs and lung SCCs. Small cell lung carcinoma presented a significant correlation between the pathological and immature type of blood vessels. 


\section{References}

1. Jemal A, Siegel R, Ward E, Hao Y, Xu J, Murray T and Thun MJ: Cancer statistics, 2008. CA Cancer J Clin 58: 71-96, 2008.

2. deMello DE and Reid LM: Embryonic and early fetal development of human lung vasculature and its functional implications. Pediatr Dev Pathol 3: 439-449, 2000.

3. Parera MC, van Dooren M, van Kempen M, de Krijger R, Grosveld F, Tibboel D and Rottier R: Distal angiogenesis: a new concept for lung vascular morphogenesis. Am J Physiol Lung Cell Mol Physiol 288: L141-L149, 2005.

4. Folkman J: What is the evidence that tumours are angiogenesis dependent? J Natl Cancer Inst 82: 4-6, 1990.

5. Nagy JA, Chang SH, Shih SC, Dvorak AM and Dvorak HF: Heterogeneity of the tumor vasculature. Semin Thromb Hemost 36: 321-331, 2010.

6. Carmeliet P and Jain RK: Molecular mechanisms and clinical applications of angiogenesis. Nature 473: 298-307, 2011.

7. Eberhard A, Kahlert S, Goede V, Hemmerlein B, Plate KH and Augustin HG: Heterogeneity of angiogenesis and blood vessel maturation in human tumors: implications for antiangiogenic tumor therapies. Cancer Res 60: 1388-1393, 2000.

8. Passalidou E, Trivella M, Singh N, Ferguson M, Hu J, Cesario A, Granone P, Nicholson AG, Goldstraw P, Ratcliffe C, et al: Vascular phenotype in angiogenic and non-angiogenic lung non-small cell carcinomas. Br J Cancer 86: 244-249, 2002.

9. Guo J, Higashi K, Ueda Y, Oguchi M, Takegami T, Toga H, Sakuma T, Yokota H, Katsuda S, Tonami H and Yamamoto I: Microvessel density: correlation with 18F-FDG uptake and prognostic impact in lung adenocarcinomas. J Nucl Med 47: 419-425, 2006.

10. Kakolyris S, Giatromanolaki A, Koukourakis M, Kaklamanis L, Kouroussis CH, Bozionelou V, Georgoulias V, Gatter KC and Harris AL: Assessement of vascular maturation in lung and breast carcinomas using a novel basement membrane component, LH39. Anticancer Res 21: 4311-4316, 2001.
11. Mountain CF: Revisions in the International System for Staging Lung Cancer. Chest 111: 1710-1717, 1997.

12. Sandler A, Gray R, Perry MC, Brahmer J, Schiller JH, Dowlati A, Lilenbaum R and Johnson DH: Paclitaxel-carboplatin alone or with bevacizumab for non-small-cell lung cancer. N Engl J Med 355: 2542-2550, 2006.

13. Gee MS, Procopio WN, Makonnen S, Feldman MD, Yeilding NM and Lee WM: Tumor vessel development and maturation impose limits on the effectiveness of anti-vascular therapy. Am J Pathol 162: 183-193, 2003.

14. Maeda R, Ishii G, Ito M, Hishida T, Yoshida J, Nishimura M, Haga H, Nagai K and Ochiai A: Number of circulating endothelial progenitor cells and intratumoral microvessel density in non-small cell lung cancer patients: differences in angiogenic status between adenocarcinoma histologic subtypes. J Thorac Oncol 7: 503-511, 2012.

15. Dagnon K, Heudes D, Bernaudin JF and Callard P: Computerized morphometric analysis of microvasculature in non-small cell lung carcinoma. Microvasc Res 75: 112-118, 2008.

16. Carmeliet P and Jain RK: Angiogenesis in cancer and other diseases. Nature 407: 249-257, 2000.

17. Zhao YY, Xue C, Jiang W, Zhao HY, Huang Y, Feenstra K, Resau JH, Qian CN and Zhang L: Predictive value of intratumoral microvascular density in patients with advanced non-small cell lung cancer receiving chemotherapy plus bevacizumab. J Thorac Oncol 7: 71-75, 2012.

18. Gridelli C, Maione P, Rossi A and De Marinis F: Treatment of non-small cell lung cancer: current indications and future developments. Oncologist 12: 1183-1193, 2007.

19. Jubb AM, Cesario A, Ferguson M, Congedo MT, Gatter KC, Lococo F, Mulè A and Pezzella F: Vascular phenotypes in primary non-small cell lung carcinomas and matched brain metastases. Br J Cancer 7: 1877-1881, 2011. 